\title{
LE DROIT FRANÇAIS ET EUROPÉEN DE LA CONSOMMATION
}

THE FRENCH AND THE EUROPEAN CONSUMER LAW

Vincent Lamanda*

\begin{abstract}
Résumé:
L'article donne un aperçu des relations avec les consommateurs, à travers de une analyse historique détaillée, sans oublier le concept toujours controversé de la consommation et l'influence de le «consomerism», tout en étant organisée mouvement social dans les directives européennes et la loi française.
\end{abstract}

Mots-clés: Notiondeconsommateur. Les directives européennes des consommateurs. La protection des consommateurs en droit français.

\begin{abstract}
Resumo:
O artigo oferece uma visão das relações de consumo, por meio de minuciosa análise histórica, sem olvidar do sempre controvertido conceito de consumidor e da influência do consumerismo, enquanto movimento socialmente organizados, nas diretivas européias e no ordenamento jurídico francês.

Palavras-Chave: Conceito de consumidor. Diretivas Européias de Consumo. Proteção ao consumidor no ordenamento jurídico francês.
\end{abstract}

\begin{abstract}
:
The article offers a detailed historical analysis of consumer relation, which includes an approach of the controversial concept of consumer, and the influence of consumerism, as a socially organized movement, in the European directives and the French legal system.
\end{abstract}

Keywords: Consumer concept. Europeans Directives on Consumption. Consumer protection in the French legal system.

\section{L'affaiblissement progressif du principe de l'autonomie de la volonté}

La doctrine classique, qui a dominé le droit civil pendant une partie du XIXème siècle, se rattachait à la théorie libérale qui reconnaissait comme principe essentiel la liberté des individus et leur indépendance les uns à l'égard des autres. Souhaitant rompre avec l'Ancien Régime, le droit intermédiaire, puis le Code civil affirment la liberté et l'égalité des citoyens. Les privilèges sont abolis tandis que l'ensemble des individus est soumis à une loi unique. La capacité juridique de chaque citoyen est considérée comme identique à celle de son prochain. Les corporations et les entraves religieuses, vécues comme des limitations à la liberté contractuelle, sont supprimées.

\footnotetext{
* Premier président de la Cour de Cassation.
} 
Le principe de l'autonomie de la volonté, formé des deux mots grecs, autos (soi-même), et nomos (la loi), proclamé à l'article 1134 du Code civil, fait de la volonté des parties la source essentielle du droit des obligations. Le contrat, expression de la volonté de l'individu, est élevé au même rang que la Loi, expression de la volonté collective.

Contre la théorie Kelsénienne de l'identité de l'Etat et du droit, une autre opinion tend ainsi à s'affirmer selon laquelle, non seulement l'Etat ne saurait se réduire au droit, mais encore que le droit existe aussi en dehors de l'Etat. ${ }^{1}$ Un juriste de l'époque, Fouillée, en concluait que qui dit contractuel dit juste.

Ainsi, le principe de la liberté des conventions est affirmé, sous les seules réserves des lois impératives et d'ordre public qui sont réduites au minimum. L'intervention de l'Etat dans le domaine économique est par conséquent cantonnée. Le contenu des contrats n'est plus systématiquement soumis à son approbation et le juge ne peut intervenir pour en modifier les termes.

Pour être reconnus légitimes, ces principes fondateurs reposent sur l'idée rousseauiste de l'existence d'un contrat social auquel chaque individu vient adhérer librement et sans contrainte. La liberté et l'égalité juridiques font écho à la liberté et l'égalité de fait dont chaque individu a été doté par la nature.

Ce postulat domina sans partage la pensée juridique du XIXeme siècle. Il faudra en attendre la fin pour que, sous l'influence des doctrines sociales et de la pression politique, le caractère fictif de l'égalité entre les hommes soit reconnu et que la liberté contractuelle soit considérée comme un piège pour le plus faible. ${ }^{2}$ Progressivement, et notamment sous l'impulsion de Napoléon III, auteur de l'Extinction du paupérisme, ${ }^{3}$ s'est affirmée l'idée selon laquelle l'Etat pouvait intervenir entre les parties pour protéger le plus faible. Selon la formule de Lacordaire Entre le fort et le faible, entre le riche et le pauvre, c'est la loi qui affranchit et la liberté qui opprime". Ainsi, contrairement aux postulats libéraux du XIXème siècle, on exige qu'une certaine justice règne dans le contrat lorsque l'on parle de justice contractuelle car tout ce qui est contractuel n'est pas nécessairement juste.

Le droit du travail, historiquement considéré comme une branche du droit civil consacrée à l'étude des articles 1780 et 1781 du Code civil, fut assurément le premier à s'émanciper pour constituer une discipline autonome du droit privé. Sa finalité étant d'assurer un équilibre entre deux parties, il a engendré des règles propres dont l'objet fut d'assurer la protection de l'une des parties au contrat, en général la plus faible, en l'espèce le salarié.

CADIET, L. Découvrir la justice. Paris: Dalloz Orientation, 1997. p. 65.

WEILL, Alex; TERRÉ, François. Droit civil les obligations. Précis Dalloz n. 60.

V. à ce sujet l'article de MILZA, P. La politique économique de Napoléon III. In: Napoléon III. Paris: Perrin, 2004. p 386 et s. 
2. L'affirmation des droits du consommateur dans une société postindustrielle

Le passage d'une société industrialisée à une société de consommation développa, à partir des années 1960, l'idée qu'il était nécessaire de protéger une nouvelle catégorie de cocontractants. Cette approche nouvelle, le consumérisme, vit le jour aux Etats Unis sous l'influence d'auteurs comme Herbert Marcuse, ${ }^{4}$ John Kenneth Galbraith ${ }^{5}$ et certains groupes de lobbies. ${ }^{6}$ Il fut rapidement repris par politique. Ainsi, dans son célèbre discours sur l'état de l'Union prononcé le 15 mars 1962, le Président Kennedy devait reconnaître aux consommateurs des droits positifs sur les professionnels: droit à la sécurité, droit à l'information et droit d'expression. ${ }^{7}$

En France, il fallu attendre le début des années soixante dix pour qu'apparaisse un véritable droit de la consommation, qui, prenant définitivement acte de l'inégalité contractuelle, ait pour finalité de protéger la partie la plus faible du contrat, le consommateur.

Cette époque correspondait à un développement économique sans précédent, multipliant les biens et les services proposés aux consommateurs et améliorant globalement leur qualité. Pourtant, la relation entre professionnel et consommateur apparait déséquilibrée. La compétence du professionnel, les informations dont il dispose, et souvent sa dimension financière, la complexité plus grande des produits, le développement du marketing et de la publicité, lui permettent, sinon de dicter sa loi au consommateur, à tout le moins d'avoir une emprise certaine sur lui à l'image de celle de l'employeur sur son salarié. ${ }^{8}$

Pour améliorer la protection du consommateur et rééquilibrer les relations entre les parties, le législateur et la jurisprudence admettront progressivement des atteintes à la liberté contractuelle des acteurs économiques et mettront à la charge des professionnels des obligations non expressément stipulées au contrat. Le dirigisme économique et la dépréciation monétaire contribueront également à légitimer l'intervention de l'Etat dans la sphère contractuelle.

3. L'insuffisante protection du consommateur par le droit commun des contrats

Certes, le droit civil n'avait pas attendu l'arrivée de ce droit nouveau pour prendre en compte la spécificité du consommateur et chercher à rééquilibrer les

L'homme unidimensionnel: essai sur l'idéologie de la société industrielle avancée. Traduit de l'anglais par 1964, traduction française de Monique Wittig, Les Editions de minuit.

L'ère de l'opulence, 1958, traduction française publiée en 1961 chez Calmann-Lévy.

6 tel celui de R. NADER.

7 WIEVORKA, M. Justice et Consommation. La Documentation Française, 1976.

8 CALAIS-AUlOY, Jean; STEINMETZ, Frank. Droit de la consommation. 6. ed. Paris: Précis Dalloz, s. d. n. 1. 
relations contractuelles en compensant la faiblesse ou l'ignorance de ce dernier face au professionnel..$^{9}$ Les tribunaux s'étaient en effet efforcés de remédier à la faiblesse des consommateurs lors de la souscription du contrat, notamment à travers une conception extensive de la théorie des vices du consentement.

La jurisprudence avait aussi admis l'annulation pour dol la vente souscrite par un professionnel qui passe sous silence un fait qui, s'il avait été connu de son cocontractant, l'aurait empêché de contracter, ${ }^{10}$ par exemple, la dissimulation par le vendeur d'un immeuble d'une installation prochaine d'une porcherie à proximité, ${ }^{11}$ d'un projet d'urbanisme, ${ }^{12}$ d'un projet d'élargissement d'une route, ${ }^{13}$ ou du déclenchement de la révision du plan d'occupation des sols, ${ }^{14}$ la dissimulation par un garagiste que la voiture qu' il vendait indiquait un kilométrage bien inférieur au kilométrage réel, ${ }^{15}$ ou de son état réel. ${ }^{16}$

C'est aussi pour compenser l'inexpérience ou l'ignorance du consommateur que la jurisprudence a souvent eu recours au "forçage" du contrat pour ajouter à la charge de la partie la plus forte des obligations non expressément prévues au contrat dans l'intérêt de l'usager ou du consommateur. ${ }^{17}$

Ainsi peut-on citer l'obligation de sécurité qui pèse sur le professionnel qui reçoit son client dans ses locaux ou l'obligation de renseignement et de conseil qui lui impose d'éclairer le consommateur sur la portée de ses engagement, quitte à le dissuader de contracter si le contrat risque de se révéler désavantageux pour lui. ${ }^{18}$

Ces solutions tirées du Code civil se sont cependant révélées insuffisantes pour assurer une complète protection du consommateur, et ce, pour trois raisons principales:

$1^{\circ}$ ) Elles sont insuffisantes tout d'abord parce qu'elles reposent sur l'action du consommateur. C'est à lui, s'il veut faire valoir ses droits, de prendre l'initiative d'une action judiciaire. Or, l'enjeu du litige, souvent très faible pour ce qui est des litiges de consommation, l'aléa judiciaire, la

CALAIS-AULOY, Jean. L'influence du droit de la consommation sur le droit civil des contrats. Revue Trimestrielle de Droit Civil, Paris, v. 93, n. 2, p. 239-54, avr./juin. 1994.

10 Cass Civ 3eme 15 janvier 1971, Bull Civ III n. 38, RTDCiv 1971839 obs Lousouarn, Cass Civ I 3 juin 1971 Bull Civ I n. 182 p 152, Cass Civ I 19 janvier 1977, Bull In. 40 p 30, Cass Com 21 mars 1977 JCP 1977 IV 135.

113 eme civ 2 oct 1974, Bull Civ III 330.

12 CA Amiens 4 juin 1975, D 1976, 124.

13 Cass Civ 1ere 13 février 1967, Bull Civ n. 58.

14 Cass Civ 3eme 27 mars 1991, Bull Civ I n. 58, D 1992, Somm 196 obs Paisant, RTDCiv 1992, 81, obs Mestre.

15 Cass Civ 1ere 19 janvier 1977, Bull Civ I p. 30.

16 Cass Civ 1ere 19 juin 1985, Bull Civ, I, n. 201.

17 CARBONNIER, J. Droit civil: les obligations. Paris: PUF, 2000. n. 295. (Themis)

18 Par exemple pour le garagiste qui doit avertir son client du caractère inopportun des réparations envisagées quand leur coût prévisible dépasse la valeur vénale du véhicule Cass Com 25 fevr 1981, D 1981 IR p 278, CA Rouen 18 mai 1973, JCP 1874 II 17867 note Gross, Cass Com 17 novembre 1987, ou le banquier qui doit avertir son client non-averti: ch. Mixte 29 juin 2007 (JCP 2007 n. 36 note A. Gourio, J Djoudi, F. Boucard, La protection de l'emprunteur profane, D 2008, Chron p. 500. 
complexité procédurale, les lenteurs de la justice font hésiter le plaignant à intenter son action.

$2^{\circ}$ ) Les solutions du droit civil font généralement reposer la charge de la preuve sur le consommateur. C'est à lui qu'il appartient de démontrer l'existence du vice du consentement ou de la faute contractuelle du professionnel. Or, très souvent, cette preuve est très difficile à rapporter. Comment, par exemple, démontrer l'existence de promesses verbales non tenues ou d'un abus de faiblesse?

$3^{\circ}$ ) La mise en œuvre des solutions du droit civil est souvent inadaptée à la protection du consommateur. Par exemple, selon l'article 1110 du Code Civil, l'erreur commise par une partie au contrat ne peut justifier la nullité des conventions si elle porte sur la valeur de l'objet du contrat ${ }^{19}$ ou les motifs qui ont conduit les parties à contracter. ${ }^{20}$

On en a donc conclut que le législateur devait intervenir pour équilibrer les rapports entre professionnels et consommateurs et poser des règles plus efficaces que celles du code civil.

\section{Caractères et traits dominants du droit de la consommation}

La consommation est un phénomène de masse: les intérêts, souvent petits si on les examine un à un, sont considérables dès lors qu'on les prend globalement, dans l'espace et dans le temps. ${ }^{21}$ Ces règles nouvelles doivent par conséquent résulter d'une approche différente de celle du droit civil.

Elles doivent d'abord être préventives, pour supprimer les germes d'un éventuel conflit et garantir aux consommateurs une réelle protection sans avoir à intenter un procès. L'indemnisation du préjudice, dont la mise en œuvre a un coût humain et financier parfois élevé, n'est pas la panacée pour le consommateur, d'autant que la plupart des litiges portent sur des sommes modestes: le consommateur cherche avant tout un produit ou un service conforme à son attente et répondant à tous les critères de sécurité.

Aussi, à la différence du droit civil qui intervient a posteriori pour protéger le débiteur, le droit de la consommation tend à guérir avant de réparer.

19 Cass Com 26 mars 1974, Bull Civ IV n. 108.

20 Cass Civ 3 août 1942, D.A. 1943, 18 Cass Civ 1ere 13 novembre 1990, Bull Civ I n. 242 D 1991, Somm 385 obs Aynes, RTDCiv 1991, 149, obs Bandrac.

21 CALAIS-AUlOY, Jean; STEINMETZ, Frank. Droit de la consommation. 6. ed. Paris: Dalloz, 2003. n. 2. 
On cherchera, par exemple, à retirer du marché les produits dangereux avant qu'ils ne causent des dommages, ou encore à éliminer les clauses abusives avant que les contrats ne soient conclus.

Dans le même ordre d'idée, le droit de la consommation visera à améliorer le consentement du consommateur au moment de la formation du contrat, en obligeant le professionnel à l'informer ou en lui accordant un délai de réflexion ou de repentir.

A cet égard, les règles du code de la consommation sont souvent assorties de sanctions pénales destinées à dissuader les professionnels de les enfreindre, de peur d'encourir une peine sans commune mesure avec l'avantage qu'ils pourraient en retirer.

Ainsi, un banquier préfèrera éviter de soumettre à l'emprunteur une offre de crédit non-conforme au modèle-type imposé par la loi, plutôt que d'être déchu du droit aux intérêts et d'être condamné à une amende de 30.000 euros.

De même, le publicitaire sera encouragé à vérifier la véracité de son message publicitaire pour éviter d'être condamné à une peine de deux ans d'emprisonnement et à une peine d'amende dont le montant peut être porté à 50\% des dépenses engagées pour la publicité.

Le droit de la consommation est, pour une part importante, le droit des contrats passés entre professionnels et consommateurs. Sa finalité consiste à rétablir un équilibre menacé de fait par la puissance économique du professionnel au moyen de dispositions d'ordre public, c'est-à-dire de règles auxquelles il n'est pas possible de déroger par des conventions particulières.

En effet, si l'on permettait aux parties d'écarter les dispositions protectrices de la consommation, comme c'est souvent possible en droit civil, une telle dérogation deviendrait vite une clause de style. ${ }^{22}$

Ces dispositions doivent s'appliquer même si le consommateur, qu'elles sont sensées protéger, ne les invoque pas lui-même. C'est la raison pour laquelle le code de la consommation précise que le juge peut soulever d'office toutes les dispositions qu'il contient dans les litiges nés de leur application (art. L 141-4). ${ }^{23}$

Le code civil a une approche individuelle des problèmes juridiques. Les acteurs juridiques sont appréhendés dans leurs relations individuelles avec autrui. Le droit de la consommation, tout en ne négligeant pas la relation individuelle entre le consommateur et le professionnel, va au delà pour prendre en compte la dimension collective de la consommation. Aussi, favorise-t-il le regroupement des consommateurs en associations pour leur donner davantage de force et rompre l'isolement dont ils peuvent souffrir, même si, jusqu'à présent, l'action de groupe n'est pas encore prévue.

\footnotetext{
22 PICOD, Y.; DAVO, H. Droit de la consommation. Paris: Armand Colin, 2005. n. 22.

23 Cass. 1ere civ 22 janvier 2009, pourvoi n. 0520176 et 0615370.
} 
Le droit de la consommation permet l'intervention d'organismes administratifs, comme le Conseil national de la consommation, la commission nationale des clauses abusives, la commission de sécurité des consommateurs ou la direction générale de la concurrence, de la consommation et de la répression des fraudes, organismes chargés par la loi à concourir à l'intérêt collectif des consommateurs.

5. Le périmètre du droit de la consommation

Deux conceptions du droit de la consommation peuvent être proposées:

- l'une, se fondant sur un critère matériel, considère que le droit de la consommation rassemble l'ensemble des règles applicables aux actes de consommation,

- l'autre, se fondant sur un critère finaliste, estime que le droit de la consommation est l'ensemble des règles qui ont pour but de protéger le consommateur face au professionnel. ${ }^{24}$

Le code de la consommation, promulgué en 1993, adopte une voie médiane.

Ce code rassemble dans un texte unique non seulement l'ensemble des textes destinés à protéger les consommateurs, mais aussi des dispositions du droit commun qui contribuent également à la protection des consommateurs (ainsi, l'article L 2211-1 reprend les articles 1641 et suivants du code civil relatifs à la garantie des vices cachés) et des dispositions issues du droit de la concurrence et de la distribution, et donc édictées dans le but d'assurer une concurrence loyale entre professionnels mais qui, comme par exemple la prohibition de pratiques commerciales illicites, bénéficient en fin de compte au consommateur.

Contrairement à la classification traditionnelle qui se base sur la nature des règles pour distinguer les différentes branches du droit, le droit de la consommation fait appel à une méthode plus récente qui se fonde sur la finalité des règles. Il regroupe ainsi, de façon transversale, l'ensemble des règles qui ont pour objet ou pour effet la protection du consommateur. Sa délimitation passe donc par la définition du consommateur.

\section{La notion de consommateur}

La notion de consommateur est familière depuis longtemps aux économistes. Pour ceux-ci, le consommateur est l'utilisateur final des biens et des services produits par une

${ }^{24}$ CAlAiS-AUlOY, Jean; STEINMETZ, Frank. Droit de la consommation. Paris: Précis Dalloz. n. 14. 
autre catégorie d'acteurs économiques, les producteurs. Il consomme pour la satisfaction de ses besoins personnels, alors que le producteur agit dans le but d'accroître sa richesse.

Elle ne fait pas l'objet du même consensus chez les juristes. Bien que fréquemment utilisée depuis trente ans, elle ne repose, en droit français, sur aucune définition légale, ce qui, bien entendu, n'a pu que contribuer à alimenter une longue controverse doctrinale et jurisprudentielle. ${ }^{25}$

La promulgation d'un code de la consommation aurait pu sans nul doute être l'occasion d'élaborer une fois pour toutes une définition générale. ${ }^{26} \mathrm{Il}$ n'en n'a rien été puisque la loi de 1993 qui l'a promulgué s'est contentée de compiler dans un code unique diverses dispositions législatives déjà existantes. ${ }^{27}$

La démarche tendant à rechercher l'existence d'un concept unique de consommateur est rendue d'autant plus difficile que les textes qui y font référence n'en donnent aucune définition positive. C'est davantage par les exceptions qu'ils édictent pour limiter leur champ d'application que se dessinent les contours de la notion de consommateur.

L'absence de définition précise a bien évidemment fait tâtonner la jurisprudence de la Cour de cassation dans cette démarche.

Un certain nombre d'hypothèses ne posent pas de difficulté. Un particulier qui acquiert un meuble destiné à son domicile personnel est sans nul doute un consommateur. A l'opposé, un commerçant qui achète une marchandise pour la revendre n'est pas un consommateur. Les difficultés se manifestent, comme toujours, aux frontières, c'est à dire

25 CORNU, Gerard. Rapport sur la protection du consommateur et l'exécution du contrat en droit français, Trav. Assoc H. CAPITANT t 24, 1973, Dalloz 1975 p. 131; BERLIOZ, G. Droit de la consommation et droit des contrats, JCP 1979, ed. G, I, 2954; ROUHETTE, G. Droit de la Consommation et théorie générale du contrat, Et. Rodière, Dalloz 1981, p. 247-272, PIZZIO, J. P. L'introduction de la notion de consommateur en droit français, D 1982, chr 91; rep com Dalloz, Droit de la consommation, 1987, n. 416; MALINVAUD, Ph. La protection du Consommateur; D 1981 Chr 49 n. 2; Le Consommateur, 81 eme Congrès des Notaires de France, Lyon 1985; Malaurie, Le Consommateur, Def 1985, art 33593; CAS, G.; FERRIER, D. Traité de droit de la Consommation, PUF 1986, n. 8; GHESTIN, Jacques. Le Contrat, LGDJ 1988, n. 59; OILLICLEPETIT, A. La Notion de consommateur en droit français; Contrats-Conc-Consom, 1988, n. 44, p. 3; MESTRE, J. Des notions de Consommateurs, RTD Civ 1989, p. 62s, GIAUME, Ch. Le non professionnel est-il un consommateur? où les problèmes de la redondance en droit de la consommation: Petites Affiches 23 juillet 1990 p 25; CALAIS-AULOY, J.; STEINMETZ, F. Droit de la Consommation, Dalloz 4eme ed 1996, n. 9; PAISANT, G. Essai sur la notion de consommateur en droit positif, JCP ed G 1993 3655, MARTIN, L. R. Le Consommateur Abusif, D 1987, Chr p. 150, H Davo, Fasc Jurisclasseur Civil, Contrat et Obligation, Protection du Consommateur, 1992, art 1109 fasc 10; RAYMOND, G. Commentaire de la loi du 18 janvier 1992 renforçant la protection des consommateur, Contrat, Concurrence, Consommation février 1992; SINAY-CYTERMANN, Protection ou surprotection du consommateur, JCP ed G 1994, Doctr 3804.

26 CALAIS-AULOY, Jean. Proposition pour un nouveau droit de la consommation. Paris: La Documentation Française, 1985; Proposition pour un code de la documentation. Paris: La Documentation Française 1990.

27 RAYNAUD, J. L. A propos du Code de la Consommation. GP, 26, 27 janvier 1994, n. 26, 27 Doctr p. 4, BUREAU, D. Remarques sur la Codification du droit de la consommation. Recueil Dalloz Sirey, 1994, Chron p. 291, Giverdon, Lecharny, Regnier, Verdun, GP 4 février 1994 p. 8, Code de la Consommation Commenté par J. P. PIZZIO, ed Montchretien 1995. 
dans les cas où un agent économique, bien qu'intervenant pour les besoins de sa profession, agit en dehors de sa spécialité et se trouve dans le même état d'infériorité qu'un particulier contractant pour ses besoins personnels (ce que la Doctrine définit comme étant un nonprofessionnel).

La Doctrine se prononce majoritairement en faveur d'une définition stricte, proche de sa définition économique. Pour elle, le consommateur c'est "l'acquéreur non professionnel de bien de consommation destiné à son usage personnel", 28 "la personne qui, pour ses besoins personnels, non professionnels, devient une partie à un contrat de fournitures de biens ou de services, ${ }^{29}$ la personne physique ou morale de droit privé qui se procure ou utilise des biens et des services pour un usage non professionnel. ${ }^{30}$

La question est nettement tranchée en droit communautaire en faveur de la thèse restrictive. Les textes européens, les directives ou les règlements de Bruxelles et de Rome, et la jurisprudence de la Cour de justice des communautés européennes limitent le bénéfice des dispositions consuméristes destinées à corriger le déséquilibre entre les parties aux personnes physiques contractant pour la satisfaction de leurs besoins personnels et pour un usage étranger à leur activité professionnelle. ${ }^{31}$

Après avoir, dans un premier temps et de façon isolée, reconnu la qualité de consommateur à un agriculteur démarché pour souscrire une police d'assurance relative à son exploitation, ${ }^{32}$ la Cour de cassation devait adopter ensuite la thèse soutenue par la Doctrine dominante pour refuser de reconnaître la qualité de consommateur à un agent d'assurance démarché pour réaliser une opération de publicité de son cabinet. ${ }^{33}$

28 proposé par MM Cornu et Ghestin, Trav. de l'Assoc. H. Capitant, 1973, p. 135.

29 GHESTIN, Jacques. Le Contrat. Paris: LGDJ, 1980. n. 59.

30 CALAIS-AUlOY, Jean; STEINMETZ, Frank. Droit de la consommation. Paris: Précis Dalloz. n. 3, Projet de Code de la Consommation, art L3 e CALAIS-AULOY, Jean. Propositions pour un code de la Consommation; Rapport de la commission pour la codification du droit de la consommation; Doc. Fr. avril 1990 p. 20.

31 Dir 85/577 du 20 décembre 1985 sur la protection des consommateurs dans le cas de contrats négociés en dehors des établissements commerciaux, dir 99/44 du 25 mai 1999 sur certains aspects de la vente et des garanties des biens de consommation, CJCE 21 juin 1978, Bertrand, aff 150/77, Rec CJCE p. 1431, CJCE 14 mars 1991, P. di Pinto, aff C 31/89, Rec CJCE, I, p. 1206, CJCE 3 juillet 1997, D 1997, IR p. 174, cf Monique Luby La notion de consommateur en droit communautaire: une commode inconstance; également CJCE 3eme ch, 22 novembre 2001, aff. jointes C 541/99 et C 542/99; Cape Snc c/ Idealservice et Idealservice MNRE Sas c/ Omai Srl, JCP 2002, II Jur 10047 note Gilles Paisant, Contrats, Concurrence, Consommation Chroniques n. 14 note M. Luby, Dalloz 2002, Jur p. 2929 obs J. P. Pizzio, également CJCE, 20 janv. 2005, aff.C-464/01, Gruber la personne qui a conclu un contrat portant sur un bien à usage mixte ne peut se prévaloir des règles favorables de compétence juridictionnelle prévues par la convention de Bruxelles du 27 septembre 1968 au bénéfice des consommateurs, sauf à ce qu'il soit démontré que l'usage professionnel du bien est marginal au point d'avoir un rôle négligeable dans le contexte global de l'opération en cause.

32 Cass Civ I 15 avril 1982 D 1984, J p 439 note J. P. Pizzio.

33 Cass Civ 1ere 15 avril 1986, D 1986, IR 393 obs Aubert, ou à un commerçant en appareils électro-ménager, Cass Civ I 23-06-87, Bull 87 I no 209 p. 154. 
Cette position, qui oppose le consommateur au professionnel, s'attache au but poursuivi par le consommateur, à l'objet du contrat. Le critère de distinction est donc subjectif. Le consommateur est celui qui contracte pour satisfaire ses besoins personnels à l'inverse du professionnel qui agit pour les nécessités de son activité professionnelle. Il n'est donc pas tenu compte de l'état réel d'infériorité du professionnel, qu'il soit petit artisan ou société anonyme, néophyte ou expérimenté.

A l'inverse, le professionnel qui agit pour satisfaire des besoins non professionnels est considéré comme un consommateur.

La première chambre civile de la Cour de cassation devait opérer par la suite un revirement de jurisprudence en reconnaissant à un agent immobilier faisant installer un système d'alarme dans son cabinet le droit de se prévaloir des dispositions contenues dans l'article 35 de la loi du 10 janvier 1978 relative aux clauses abusives. ${ }^{34}$ Pour rendre applicables à ce professionnel les dispositions de cette loi dont le bénéfice est réservé aux seuls consommateurs, la Cour de cassation a considéré que ce professionnel, qui agissait en dehors de sa sphère habituelle de compétence était dans le même état d'ignorance que n'importe quel autre consommateur'. La Cour devait confirmer cette position à de multiples reprises. Ainsi, elle reconnaissait le droit de se prévaloir des lois sur le démarchage et sur le crédit à une commerçante qui commande un système d'alarme pour son magasin, ${ }^{35}$ à un artisan plombier qui souscrit un contrat d'assurance juridique, ${ }^{36}$ à un agriculteur pour l'acquisition d'un extincteur. ${ }^{37}$

Dans cette logique, la première chambre civile assimilait au consommateur la personne morale, fût-elle une société commerciale, se trouvant dans le même état d'infériorité que celui du consommateur personne physique. ${ }^{38}$

Une distinction intervenait donc entre les contrats conclus pour les besoins de la profession, "actes de la profession"39 et donc exclusifs de la notion de consommateur, de ceux conclus, certes à l'occasion de la profession, mais en dehors de la sphère habituelle de compétence, "actes relatifs à la profession". ${ }^{40}$

La première chambre civile de la Cour de cassation s'écartait ainsi de l'approche économique pour adopter une vision plus sociologique de la notion de

34 Cass Civ 1ere, 28 avril 1987 D 1988, 1, note P. Delebecque; D 1987 Somm 455 obs Aubert; JCP 1987, II, 20893 note Paisant, Paisant, les nouveaux aspect de la lutte contre les clauses abusives, D 1988, Chron 253 n. 5, RTDCiv 1987.537 obs Mestre.

35 Cass Civ I 25 mai 1992, Audijuris juin 92 n. 21 p 25 note Vigneau, D 92 somm p 401, JCP I 93 n. 3655 note Paisant, D 1993, Jur p 87 note Nicolau.

36 Cass Civ I, 20 octobre 1992, JCP 93 n. 22007 Note Paisant.

37 Cass Civ I 6 janvier 1993, Audijuris avril 92 n. 30 p 24 note Vigneau, JCP 93 n. 22007 note Paisant.

38 Cass Civ 1ere, 28 avril 1987 op. cit.

39 J.L. Aubert obs D 1988 somm 407.

40 AUBERT, J. L. op. cit. 
consommateur. La situation de l'agent dans le processus économique général importe peu. Si l'utilisateur final est nécessairement un consommateur, le producteur, situé en amont, n'est pas forcément un professionnel. L'objet du contrat constitue toujours un élément essentiel mais non déterminant puisque la seule constatation qu'un contrat n'est pas conclu pour des besoins personnels n'entraîne pas l'exclusion du champ d'application des lois consuméristes. La ligne de partage entre bénéficiaires et exclus de la protection se situe au niveau de la "sphère habituelle de compétence". Le critère devient objectif. Pour reprendre l'expression utilisée dans le rapport annuel de la Cour de cassation de 1987, "le commerçant qui contracte pour les besoins de son commerce mais en dehors de ce qui est sa technicité propre peut devenir un consommateur présumé inexpérimenté". ${ }^{41}$

Cependant, à partir de la fin de l'année 1993, on a pu déceler une très nette évolution de la position de la première chambre civile de la Cour de cassation.

Après un temps d'hésitation, ${ }^{42}$ la Cour de cassation est revenue à une position plus médiane. Dans un arrêt du 24 janvier 1995, pour écarter l'application de la loi sur les clauses abusives à un contrat de fourniture d'électricité conclu entre EDF et une société d'imprimerie, elle retient que le contrat de fourniture d'électricité avait un rapport direct avec son activité professionnelle. ${ }^{43}$ Par la suite, elle écarte l'application de ce même texte à un contrat de distribution d'eau souscrit par une société industrielle, ${ }^{44}$ puis, à un contrat portant sur l'acquisition d'un logiciel de gestion de clientèle souscrit par une société commerciale au motif identique que ces dispositions "ne s'appliquent pas aux contrats de fournitures de biens ou de services qui ont un rapport direct avec l'activité professionnelle exercée par le cocontractant". ${ }^{45}$

Ainsi, elle abandonnait le critère antérieur de la sphère habituelle de compétence du professionnel pour adopter celui du rapport direct. Selon cette jurisprudence, un professionnel devait être assimilé à un consommateur lorsqu'il concluait un contrat sans rapport direct avec son activité.

Ce nouveau critère comportait des inconvénients en ce qu'il était peu précis et faisait peser une certaine insécurité juridique.

$41 \quad$ Rapport de la Cour de Cassation 1987 p. 208.

42 Cass Civ I 24 novembre 1993, JCP ed. G. 1994, II, 22334.

43 Cass Civ I 24 janvier 1995, D 1995 Jur p 327 note Paisant, Audijuris n. 54 juin 1995 p 4 note Amlon, D 1995 Sommaires Commentés p 310 obs Pizzio, JCP 1995 ed G IV 745, Contrats, Conc, consom 1995 comm 84 note Leveneur, RTDCiv 1995 p 362 obs Mestre, JCP 1005 I 3893 n. 28 obs Viney.

44 Cass Civ I 3 janvier 1996, D 1996 IR p 47, D 1996 jur p 228 note Paisant, JCP 1996 I 3929 obs Labarthe.

45 Cass Civ I 30 janvier 1996 D 1996 jur p 228 note Paisant, Audijuris n. 64 mai 1996 p 18 note Vigneau, JCP 1996 IV 673, Contrats, Conc. Consom 1996 n. 46 obs Raymond) (Position identique de la chambre criminelle: Cass Crim 29 juin 1999, BICC 15 octobre 1999 p. 8 n. 1105) (également, en dernier lieu Cass Civ I 23 février 1999, D 1999, inf rapp p. 82. 
Sensible aux critiques de la doctrine, et soucieuse de s'aligner sur la jurisprudence européenne, la Cour de cassation, dans un arrêt du 11 décembre $2008,{ }^{46}$ a récemment modifié sa jurisprudence.

Un contrat de dépôt d'un distributeur automatique de boissons avait été conclu entre deux sociétés commerciales. Une des parties ayant violé la clause d'exclusivité insérée dans ce contrat, elle assignée en résiliation judiciaire et en réparation du préjudice subi par son cocontractant. Afin d'échapper à toute responsabilité, elle invoquait le caractère abusif de la clause qui devait être, selon elle, réputée non écrite, par application de l'article L.132$1 \mathrm{du}$ Code de la consommation. Elle prétendait ainsi se placer sous le sceau protecteur du droit de la consommation malgré son statut de professionnel au motif que l'objet du contrat n'avait aucun rapport avec son activité professionnelle.

La Cour de cassation casse la décision de la cour d'appel qui avait accueilli cette argumentation en énonçant que les contrats de fournitures de biens ou de services conclus entre des sociétés commerçantes échappent au droit de la consommation.

La Cour de cassation refuse ainsi désormais d'accorder la protection aux professionnels en ne se fondant non plus sur le rapport direct avec l'activité professionnelle mais sur la qualité du cocontractant, critère déterminant pour l'application des dispositions concernant les clauses abusives. Le rapport direct, dont l'appréciation était laissée au pouvoir souverain des juges du fond, ${ }^{47}$ était par trop porteur d'insécurité juridique. La Cour de cassation opte pour une décision plus claire et sûre car dès lors qu'un contrat de fournitures de biens ou de services a été conclu entre des sociétés commerciales, ce contrat ne peut pas être considéré comme un contrat de consommation du fait de la qualité même des cocontractants.

Cet arrêt marque un abandon implicite de la notion du lien direct, abandon qui ne peut être qu'approuvé. En effet, dans tout exercice d'une activité professionnelle, il est indispensable pour une société commerciale de conclure des contrats de fourniture de biens et de services. Ces contrats sont nécessaires au bon fonctionnement de l'entreprise. De tels contrats sont, par nature, des contrats si classiques pour un professionnel qu'il ne peut revendiquer un quelconque manque de connaissance ou de pratique. Le but du droit de la consommation n'est pas de lutter contre touts les déséquilibres contractuels; c'est un droit spécial qui n'a vocation à s'appliquer que dans de stricts rapports entre professionnels et consommateurs, entendus comme une personne qui contracte pour un usage familial ou personnel, ce qui n'est pas le cas d'une société commerciale.

\footnotetext{
$46 \quad$ lere civ 11 décembre 2008 pourvoi n. 07-18128.

47 Civ $1^{\text {ère }}, 17$ juillet 1996.
} 


\section{Les différentes facettes du droit de la consommation}

Comme le droit du travail, le droit de la consommation transcende la classification traditionnelle entre droit public et droit privé, et au sein de celui-ci, droit civil, droit pénal, droit commercial. Si le droit de la consommation a pour base le droit civil puisque la plupart des actes de consommation sont des contrats civils, il emprunte également aux autres classifications:

- au droit de la concurrence: de nombreuses dispositions destinées à réglementer les rapports entre entreprises passent par une régulation des relations entre ces entreprises et leurs clients consommateurs: ${ }^{48}$ ainsi, la réglementation sur les fraudes, sur l'étiquetage, sur les soldes, sur le refus de vente, etc.

- au droit commercial: de nombreux actes de consommation trouvent leur origine dans des actes de commerce. Ainsi, le recours au crédit a d'abord été réservé aux commerçants avant d'être étendu aux consommateurs. De plus, les actes de la consommation sont généralement des actes mixtes entre commerçants et consommateurs.

- au droit pénal: de nombreuses obligations imposées aux professionnels dans le but de protéger les consommateurs sont assorties de sanctions pénales; par exemple dans la loi de 1972 sur le démarchage ou celle de 1978 sur le crédit à la consommation;

- au droit administratif: face à certains services publics, les usagers se trouvent en situation de véritables consommateurs: services des eaux, EDF. En outre, une partie du dispositif de protection du consommateur repose sur des mécanismes de droit public.

- au droit judiciaire: le souci d'assurer au consommateur un meilleur accès à la justice a conduit à des réformes de la procédure civile. Ainsi, la saisine du tribunal d'Instance par déclaration au greffe, l'injonction de faire, l'action collective des consommateurs, etc.

- au droit européen: la construction communautaire repose, entre autres, sur le principe de la libre circulation des marchandises entre Etats membres. L'article 28 du Traité de la Communauté Européenne interdit toute restriction à l'importation ou à l'exportation entre Etats membres, sauf si elles sont justifiées par des raisons de moralité ou de santé publique.

48 voir à cet effet la chronique de Jacques Amar Une cause perdue: la protection des personnes morales par le droit de la consommation in Contrats, concurrence, consommation, 1993 chron n. 5. 
Le moyen le plus simple pour parvenir à une uniformisation des conditions de mise sur le marché des produits européens consiste à établir entre Etats membres une harmonisation de leurs législations.

Dans cette optique, le Conseil des Communautés Européennes puis de l'Union européenne a été conduit à plusieurs reprises à élaborer des directives tendant à harmoniser la réglementation des rapports entre professionnels et consommateurs:, la directive du 10 décembre 1984 relative à la publicité trompeuse, la directive du 25 juillet 1985 sur la responsabilité du fait des produits défectueux, la directive du 5 avril 1993 relative à l'élimination des clauses abusives, ${ }^{49}$ la directive du 20 mai 1997 sur la protection des consommateurs dans le domaine de la vente à distance, la directive du 8 juin 2000 sur le commerce électronique. La transposition en droit interne de ces textes communautaires a contribué à l'édification d'un droit européen unifié de la consommation.

Mais lorsqu'il n'existe pas d'harmonisation européenne, la Cour de justice des Communautés Européenne considère que tout produit ou service conforme à la réglementation d'un Etat membre doit pouvoir circuler librement à l'intérieur du marché unique européen, de sorte que l'Etat d'importation ne peut s'opposer, sauf pour des motifs liés à la sécurité des consommateurs, à la commercialisation d'un produit au motif qu'il ne serait pas conforme à sa propre réglementation, dès lors qu'il est conforme à la réglementation du lieu de vente ou de production. ${ }^{50}$

La Cour de justice va jusqu'en déduire que les Etats membres ont l'obligation d'insérer dans leur réglementation nationale une clause de reconnaissance mutuelle permettant de commercialiser sur leur territoire des produits légalement commercialisés sur le territoire d'un autre Etat membre ${ }^{51}$ (par ex. pour la France l'article R 112-14-1 du code de la consommation).

Cette jurisprudence repose sur un principe de reconnaissance mutuelle des législations nationales. Comme l'a souligné le Livre blanc sur l'achèvement du marché intérieur, ce principe s'appuie sur l'idée que les réglementations nationales provenant de pays arrivés à un même stade de maturité économique doivent être considérées comme équivalentes, car elles poursuivent des objectifs semblables, même si, pour les atteindre, elles emploient des moyens équivalents. ${ }^{52}$

\footnotetext{
49 PAISANT, Gilles. Propositions pour une réforme du droit des clauses abusives, JCP 1994 I n. 3772.

50 CJCE 20 février 1979 Cassis de Dijon, Rev. Trim. Dr. Europ. 1980. 765, CJCE 25 juillet 1991 aff C/76/90 Säger et Dennemeyer Rec I p. 4221, C 288/89, Gouda, Rec I p. 4007 et C 353/89 Commission c Pays Bas, Rec I p 4069 p 4069, GP 10 au 12 décembre 1995 Jurisprudence p. 18 note Huglo, CJCE 24 mars 1994 affaire C/275/92 Schindler, Rec I p 1039, GP 10 au 12 décembre 1995 p. 21 note Huglo, CJCE 10 mai 1995, aff C 384-93Alpine Investissement, D 1995 sommaires Commentés p. 317 pour des services financiers proposés par téléphone.

51 Affaire Foie Gras CJCE 22 oct. 1998, aff. C-169/98.

52 PIZZIO, J. P. op. cit.
} 


\section{L'influence du droit de la consommation}

Le mouvement consumériste n'a pas seulement eu pour effet de contribuer à l'édification et la reconnaissance d'une nouvelle branche du droit. Il a irrigué l'ensemble des matières juridiques. Le droit civil, le droit commercial et le droit de la concurrence, pour ne citer qu'eux, ont chacun évolué dans le sens d'une meilleure prise en compte de l'inégalité économique entre les parties contractantes.

Ainsi, à titre d'exemple, l'introduction, par la loi du 2 août 2005, dans le code de commerce, de l'article L.420-2 qui prohibe, sous certaines conditions, l'exploitation abusive par une entreprise ou un groupe d'entreprises d'une position dominante sur le marché intérieur ainsi que l'état de dépendance économique dans lequel se trouve (...) une entreprise cliente ou fournisseur dés lors qu'elle peut affecter le jeu de la concurrence sur un marché. Cette législation s'inspire directement de la jurisprudence de la Cour de cassation qui avait, auparavant, admis que la faiblesse économique ou psychologique ou l'ignorance d'une partie pouvait constituer une contrainte morale de nature à caractériser un vice du consentement lorsque: l'exploitation abusive d'une situation de dépendance économique est faite pour tirer profit de la crainte d'un mal menaçant directement les intérêts légitimes de la personne. ${ }^{53} \mathrm{C}$ 'est encore une interprétation extensive de l'article 1112 du Code civil, selon lequel "la violence doit être de nature à faire impression sur une personne raisonnable, et pouvoir lui inspirer la crainte d'exposer sa personne ou sa fortune à un mal considérable qui a permis l'annulation d'un cautionnement contracté sous l'empire d'une violence morale par l'épouse d'un dirigeant de société mise en liquidation. ${ }^{54}$ C'est également le cas de l'augmentation de capital consentie par des actionnaires au profit d'une autre société sous la menace d'une mise en liquidation de leur société ${ }^{55}$ ou de l'engagement signé sous la menace d'interrompre un séjour de 140 personnes participant à un voyage à 1 'étranger. ${ }^{56}$

\footnotetext{
53 Cass Civ $1^{\text {ère }} 3$ avr. 2002, Bull Civ I n. 108, D 2002, jur p 2834 obs D. Mazeaud, D 2002, jur p 1860 note Gridel et Chazal, Contrats, conc., cons. 2002, comm n ${ }^{\circ} 121$ obs L. Leveneur, BRDA 2002, p 2 n. 12, G. Loiseau, L'éloge du vice ou les vertus de la violence économique, Dr et patrimoine 200., p 26, JCP éd G 2002, I n. 19 obs G. Virassamy.

54 Cass com 28 mai 1991, D 1992, 166 note Morvan, D 1991 Somm 383 note L. Aynes, RTD civ 1992, 85, obs J. Mestre.

55 Cass com 18 fév. 1997, Bull Civ IV n. 59, Bull Joly 1997, p 408, obs JJ Daigre, D 1998, somm p 181 obs JC Hallouin, on peut citer aussi l'arrêt de la première chambre civile qui a approuvé une cour d'appel qui avait accueilli la demande de restitution d'honoraires excessifs perçus par un avocat, au motif que l'ignorance de ce que pouvait être dans les circonstances de l'affaire le montant normal de tels honoraires avait constitué l'un des éléments de la contrainte morale ayant déterminé son client, en situation d'infériorité manifeste, à lui régler la somme demandée: Cass Civ 1 ìre, 3 nov. 1976, GP 1977, 1, 67, note Damien.

56 TGI Bourges 11 avril 1989, GP 1990, 1, Somm, 310.
} 
Le droit de la consommation a accoutumé d'imposer aux professionnels une obligation spontanée d'information à leurs cocontractants non seulement avant la formation du contrat, mais aussi pendant son exécution. On sait que, quel qu'en soit le domaine, la détention de l'information est un élément capital dans la prise de décision et que son détenteur dispose d'un pouvoir considérable sur son cocontractant. Pour éviter que le professionnel n'abuse de l'information que lui confère sa compétence pour tirer des profits illégitimes, le droit de la consommation l'oblige à la partager avec son cocontractant. Lorsque la diffusion de l'information est spontanée de sa part, il s'assure de sa loyauté. Dans les domaines qui ne faisaient pas l'objet d'une législation consumériste particulière, les magistrats de la Cour de cassation ont, de lege lata, imposé aux professionnels un partage de l'information.

C'est ainsi qu'à rebours d'une règle traditionnelle selon laquelle un client n' pas fondé à se plaindre du "bonus dolus" du vendeur résultant d'une exagération des qualités de la marchandise ou du passage sous silence de ses défauts, la jurisprudence juge désormais que le dol peut être constitué par le silence d'une partie dissimulant à son cocontractant un fait qui, s'il avait été connu de lui, l'aurait empêché de contracter. ${ }^{57} \mathrm{Il}$ a ainsi été jugé que le banquier pouvait commettre une réticence dolosive à ne pas informer la caution de la situation irrémédiablement compromise du débiteur principal et ce, même si une clause contractuelle précise que la caution ne fait pas de la situation du cautionné la condition déterminante de son engagement. ${ }^{58}$

Pour Justinien, le juge détenait la libre puissance d'exprimer combien il était dû, d'après ce qui est bien et équitable (ex bono et aequo aestimandi). ${ }^{59}$ Plus adaptée aux exigences de la vie économique actuelle où la multiplication des acteurs et la rapidité des transaction rend encore plus nécessaire l'exigence de loyauté des cocontractants, les juges modernes trouvent aussi, dans l'obligation générale de contracter de bonne foi énoncée à l'article $1134 \mathrm{du}$ code civil ou dans les maximes d'interprétation des articles 1160 (“on doit suppléer dans le contrat les clauses qui y sont d'usage, quoiqu'elles n'y soient pas exprimées") et 1135 du Code Civil ("les conventions obligent non seulement à ce qui y est exprimé, mais encore à toutes les suites que l'équité, l'usage ou la loi donnent à l'obligation d'après sa nature") la justification d'une jurisprudence qui renforce les obligations de la partie forte dans l'intérêt de la partie faible, conférant à cette dernière une véritable "cuirasse" juridique la protégeant de la puissance économique de son

\footnotetext{
57 Cass Civ 3eme 15 janvier 1971, Bull Civ III n. 38, RTD civ. 1971839 obs Y. Loussouarn, Cass Civ I 3 juin 1971 Bull Civ I n. 182 p 152, Cass Civ I 19 janvier1977, Bull I n. 40 p 30, Cass Com 21 mars 1977 JCP 1977 IV 135.

58 Cass Civ 1ere 21 janv. 1981, Bull Civ I n. 25 et Cass. Com 8 nov. 1983, Bull IV n. 298, Cass Civ 1 ere 10 mai 1989, RTD Civ 1989 obs J. Mestre et Cass. 1ère civ., 13 mai 2003: Bull. Civ. I, n. 114; D. 2004. 262, note Mazuyer; JCP 2003. II. 10144, note Desgorces et I. 270, note G. Loiseau; Defrénois 2003. 1568, obs. Libchaber; RTD civ. 2003. 700, obs J. Mestre et B. Fages.

59 LÉVY, J. CASTALDO, P. A. op. cit. n. 466 p. 691.
} 
cocontractant. ${ }^{60}$ Ces principes, pourtant issus des sources du droit les plus anciennes, mais revivifiés par la vitalité du droit de la consommation, trouvent encore, dans les exigences de la vie moderne leur pleine justification.

Paris, outubro de 2009.

60 CARBONNIER, J. Droit civil: les obligations. Paris: PUF, 2000. n. 295. (Themis) 